\title{
Pediatrics Standardized Concentration of Chemotherapy Intravenous Infusion: A New Initiative in Saudi Arabia
}

\author{
Yousef Ahmed Alomi* ${ }^{(D,}$, BSC. \\ Pharm, MSc. Clin Pharm, BCPS, BCNSP, DiBA, \\ $\mathrm{CDE}$, Critical Care Clinical Pharmacists, TPN \\ Clinical Pharmacist, Freelancer Business \\ Planner, Content Editor and Data Analyst, \\ Riyadh, Saudi Arabia. \\ Faiz A Bahadig, R.Ph, Informatics \\ Pharmacist, Pharmaceutical Care \\ Department, King Abdul-Aziz Medical, City- \\ WR-Jeddah, Ministry of National Guard, Saudi \\ Arabia. \\ Hani Alhamdan, BSc.Pharm, MSc. \\ Pharm, MBA, Director, Pharmaceutical Care \\ services Department at KAMC-Jeddah, \\ Ministry of National Guard, Saudi Arabia.
}

\section{Correspondence:}

Dr. Yousef Ahmed Alomi, Bsc. Pharm, msc. Clin pharm, bcps, bCNSP, DiBA, CDE Critical Care Clinical Pharmacists, TPN Clinical Pharmacist, Freelancer Business Planner, Content Editor and Data Analyst, P.O.BOX 100, Riyadh 11392, Riyadh, SAUDI ARABIA.

Phone no: +966 504417712

E-mail:yalomi@gmail.com
Received: 22-10-2019;

Accepted: 01-01-2020

Copyright: (c) the author(s), publisher and licensee International Journal of Pharmacology and Clinical Sciences. This is an open-access article distributed under the terms of the Creative Commons Attribution Non-Commercial License, which permits unrestricted non-commercial use, distribution, and reproduction in any medium, provided the original work is properly cited.

This is an open access article distributed under the terms of the Creative Commons AttributionNonCommercial-ShareAlike 4.0 License

Access this article online

\begin{tabular}{|c|c|}
\hline & www.ijpcs.net \\
\hline & DOI: \\
\hline
\end{tabular}

\begin{abstract}
Objectives: To discover the pediatrics and neonates standardized concentration of chemotherapy intravenous infusion as new initiatives in the Kingdom of Saudi Arabia. Methods: It is a new initiative project drove by national standardized concentration of chemotherapy intravenous infusion services. The projects formulated from the international business model, pharmacy project guidelines and project management institution guidelines of a new project. The initiative project is written through project management professionals and consisted of several parts, including the initial phase, the planning phase, the execution phase, the monitoring and controlling phase. Results: The pediatrics and neonates standardized concentration of chemotherapy intravenous infusion services with a defined vision, mission and goals. The services had various paybacks including clinical and economic on patients as discovered in the review. The continuous of the project was assured by risk management model description and the monitoring and controlling of the services as declared. The transition to operation project though closing project stage demonstrated in the analysis. Conclusion: The pediatrics and neonates standardized concentration of chemotherapy intravenous infusion services is a new initiative as part of the intravenous admixture program. The pediatrics and neonates standardized concentration of chemotherapy intravenous infusion will lessen medication errors and recover patient safety at healthcare system; it is highly recommended to implement in the Kingdom of Saudi Arabia.
\end{abstract}

Keywords: Pediatrics, Neonates, Standardized, Concentration chemotherapy, Intravenous, Saudi Arabia.

\section{INTRODUCTION}

In 2012-2015, during the implementation of the medications safety program, the pediatrics pharmacy program was part of the plan at several hospitals in the Kingdom of Saudi Arabia. ${ }^{[1,2]}$ The pediatrics or neonatal pharmacy services contained of several programs and projects. The pediatrics medications safety program was part of the plan. The pharmacist did an excellent job during this period and declared the clinical and economic outcome of medication safety in pediatrics healthcare services. ${ }^{[3,4]}$ Furthermore, the pharmacist discovered the noteworthy role in total parenteral nutrition, intervention to reduce morbidity and avoid additional superfluous costs in the healthcare system..$^{[5,6]}$ The pharmacist started standardized total parenteral nutrition in the pediatrics, neonates to prevent TPN related problems, and reduce healthcare providers and pharmacy staff workload. ${ }^{[7,8]}$ Several litterateurs' showed mistakes with chemotherapy in pediatric patients, while local or the Middle East literature not existed. ${ }^{[9,10]}$ On the other hand, the standardized concentration of chemotherapy medications is a new initiative project as expandable of the role of the intravenous pharmacist in the medications safety program with pediatrics and neonatal populations. ${ }^{[11-14]}$ The aim of the project is to review pediatrics and neonatal standardized concentration of chemotherapy medications in the Kingdom of Saudi Arabia.

\section{Method of the Project}

It is a new initiative project drove from the national IV admixture and chemotherapy program. ${ }^{[15]}$ The task force team of standardized chemotherapeutic concentration formulated and involved of from author's expert in the parenteral medications. The committee utilized and drove the pharmacy parenteral administration guidelines and from the textbook and international literature standardized concentration of chemotherapeutic written by utilizing the international business model, pharmacy project guidelines and project management institution guidelines of a new project. $^{[16-19]}$ The standardized concentration adjusted based on the acceptable concentration, daily dose and the volume of bag as possible. The project is written through project management professionals and entailed of several parts, including the initial phase, the planning phase, the execution phase, the monitoring and controlling phase.

\section{Initiative Phase}

\section{Assessment Needs}

Each intravenous admixture services prepare a daily basis for various medications with 
different concentrations and multiple diluent solutions. Also, the nurses administer different medications with different concentrations and solution. A higher workload of parenteral admixture services had been done by pharmacy staff and received by patients through nursing care. The high workload of the various number of concentration and solution may lead to the preparation or administration mistakes. As a result, the standardized concentration and solution of demand to prevent medication errors and reduce the workload of healthcare providers. Moreover, it's excellent opportunity to inspire pharmaceutical companies to manufacture the same and frequency fixed ready-made solutions.

\section{Market Analysis}

Several medications came as powers for reconstitution or ampoules for mixing with diluent solutions. Other medications came as ready-made solution or diluted with a solution ready for administration. The ready came as strength of medications regardless of the approbate concentration administration through a central venous or peripheral line. Also, the pharmacy services had a manual preparation of parenteral medications with various concentration and solution diluted in. Those multiple factors may progress to increase workload and medications mistake. As a result, the standardized concentration of intravenous medications might decrease the workload and advance medication safety in practice.

\section{SWOT Analysis}

The most popular method utilized in quality management called the SWOT Analysis, which stands for the strengths, weaknesses and opportunities elements. The project had several strong points; for instance, implemented medication safety prevention measures, the reductions of the pharmacy with healthcare workload reductions. On the other hand, the weak points, including a few medications concentration and a limited number of diluent solutions. Moreover, there are multiple points for the opportunity; for instant implementation of patient safety and accreditation standards processes, while the threat points are if the higher administration of the project or pharmacy plan changes in the future.

\section{Planning Phase}

\section{Scope of the Project}

The project covers the pediatrics and neonatal standardized chemotherapy concentration based on drug dosing and frequency administration. Besides, the concentration used as a common and maximum one with one to two solution dilution of chemotherapy with excellent stability.

\section{Vision, Missions, Goals}

The vision of the project is best pediatrics and neonatal standardized intravenous chemotherapy concentration. The message of the project is to provide suitable standardized intravenous chemotherapy concentrations for pediatrics and neonates with high stability and diluent solution. The aim of the project is to standardize the concentration of intravenous chemotherapy for pediatrics and neonates to prevent any drug concentrationrelated problems, diminution the workload of healthcare providers and pharmacy staff and prevent the economic burden of the healthcare system of medication wastage.

\section{Project Description}

The following policies were put in place for every pharmacist and other health care individuals:

$\checkmark$ The pediatrics and neonatal standardized chemotherapy concentration committee should be formulated at healthcare organizations.

$\checkmark$ The pediatrics and neonatal standardized chemotherapy concentration committee should consist of pediatrics, neonate's IV pharmacist, pharmacy technician, pediatric oncology nursing representative, pediatric oncology surgical or medical representative, pediatrics physician, nurse representative, neonatal physician and nurse representative.

$\checkmark$ The committee revises the pediatrics and neonatal standardized chemotherapy concentration and informs at least annually.

$\checkmark$ The education and training sessions should be conducted by the committee to all healthcare providers, including oncology physicians and nurses, with pediatrics and neonate's pharmacy staff.

$\checkmark$ The pediatrics and neonatal standardized chemotherapy concentration distributed to healthcare sectors at the institutions (Table 1).

$\checkmark$ The physician transcribes the prescription based on the pediatrics and neonatal standardized chemotherapy concentration.

$\checkmark$ If the physician wishes to prescribe outside the pediatrics and neonatal standardized chemotherapy concentration guidelines, he should document the justification. $\checkmark$ The prescription should send to the pharmacy, pediatrics IV pharmacist and pharmacy technician will prepare it based on the pediatrics and neonatal standardized chemotherapy concentration.

$\checkmark$ The pharmacy staff sends the medications to the nursing department, and the nurse administers the medications based on the standardized concentration of medication guidelines.

$\checkmark$ The pharmacy department should measure the clinical outcome of the pediatrics and neonatal standardized chemotherapy concentration.

$\checkmark$ The pharmacy department should measure the economic outcome of the pediatrics and neonatal standardized chemotherapy concentration.

$\checkmark$ The pharmacy department should document any prescription non-adherence to the pediatrics and neonatal standardized chemotherapy concentration.

\section{Plan Cost Management}

The financial budget should be selected in every new project. Also, the budget comprehends several things included the cost of the administration team meeting, educational courses and updated references. However, the budget should be observed from time to time.

\section{Executing Phase}

\section{Management Team}

Each project had to have a leading administrative team. The team comprised of several essential memberships' specialties. For an instant, a pediatric oncology clinical pharmacist, oncology distributive pharmacist, pharmacy technician, pharmacy total quality pharmacist, pediatrics medications safety pharmacist and pediatric oncology physician. The team had responsibilities for the implementation and monitoring of the project. The team had to educate and train concern healthcare providers about the project. The team update all standardized concentration medications list periodically and resolve any project problem-related issues until the project become one of operation system in healthcare organizations.

\section{Education and Training}

Any new project needs orientation for management team members, education, and training for pharmacy staff, including pharmacists, pharmacy technicians and the healthcare providers, including physicians and nurses. Regular orientation for the project 


\begin{tabular}{|c|c|c|c|c|c|c|c|c|c|c|c|c|c|c|c|c|}
\hline & 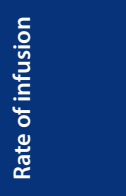 & 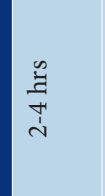 & 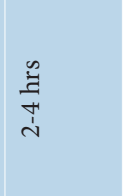 & $\begin{array}{l}\text { 节 } \\
0 \\
0 \\
\vdots \\
0\end{array}$ & & 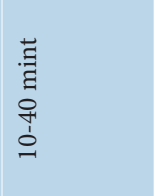 & $\begin{array}{l}\text { 音 } \\
0 \\
0 \\
0 \\
0\end{array}$ & $\begin{array}{l}\text { 音 } \\
\stackrel{0}{0}\end{array}$ & $\begin{array}{l}\text { ही } \\
\text { हू }\end{array}$ & & 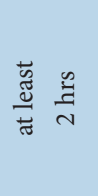 & & 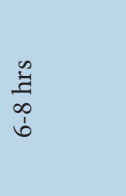 & 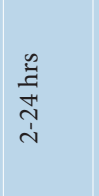 & 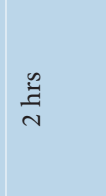 & \\
\hline & 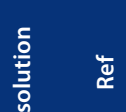 & $\frac{7}{\infty}$ & $\begin{array}{l}\vec{z} \\
\substack{7 \\
+\infty}\end{array}$ & $\overleftrightarrow{z}$ & & $\mathbb{z}$ & $\frac{m}{\infty}$ & $\overleftrightarrow{z}$ & $\overleftarrow{z}$ & & $\underset{\Xi}{\Xi}$ & & 苍 & $\frac{\Xi}{\mathbb{N}}$ & 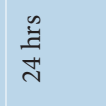 & \\
\hline & 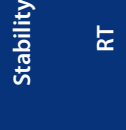 & $\frac{7}{\infty}$ & 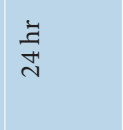 & $\overleftrightarrow{z}$ & & 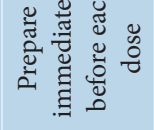 & $\mathbb{z}$ & $\frac{\ddot{J}}{\vec{J}}$ & $\frac{z}{\infty}$ & & $\frac{\vec{Z}}{\infty}$ & & $\stackrel{\vec{J}}{\underset{J}{J}}$ & $\frac{\overrightarrow{7}}{\infty}$ & $\overleftrightarrow{z}$ & \\
\hline & 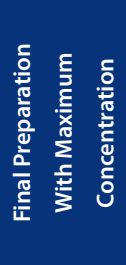 & 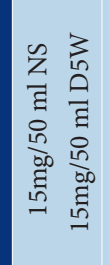 & 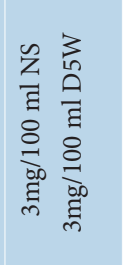 & 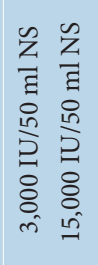 & 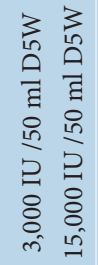 & 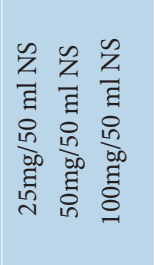 & 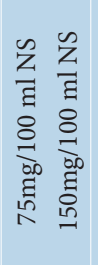 & 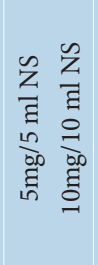 & 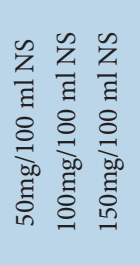 & 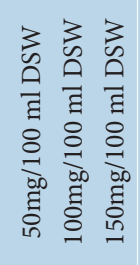 & 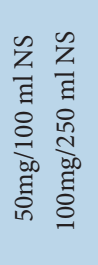 & 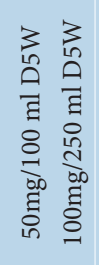 & 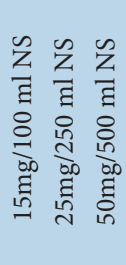 & 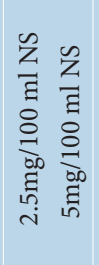 & 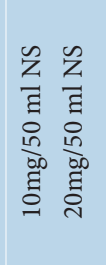 & 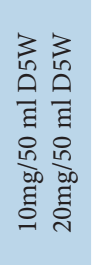 \\
\hline 患 & 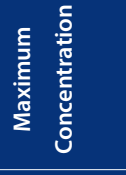 & $\begin{array}{l}\vec{\Xi} \\
\text { हो } \\
\Xi \tilde{~} \\
0 \\
0\end{array}$ & 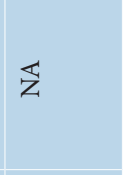 & z & & & 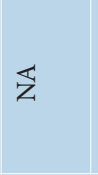 & 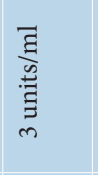 & 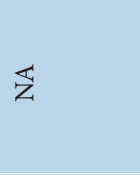 & & है & & 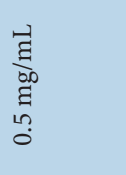 & 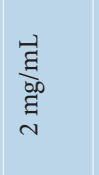 & 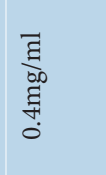 & \\
\hline 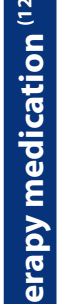 & 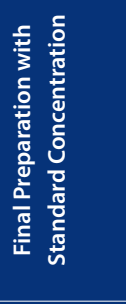 & 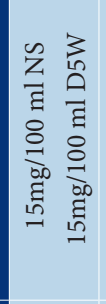 & 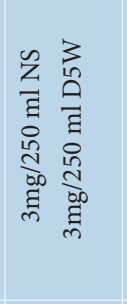 & 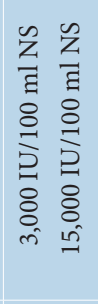 & 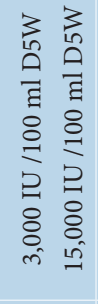 & 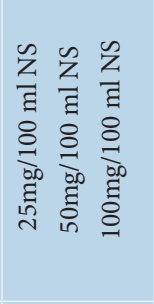 & 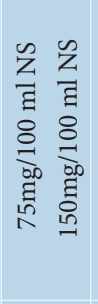 & 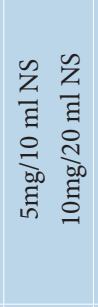 & 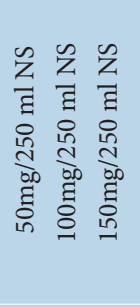 & 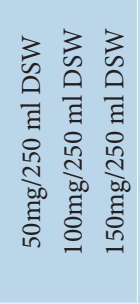 & 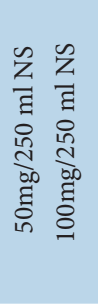 & 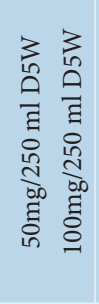 & 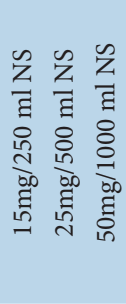 & 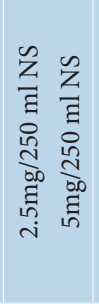 & 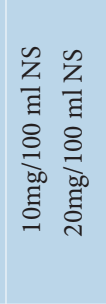 & 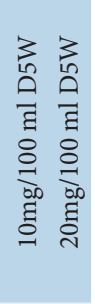 \\
\hline 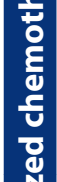 & 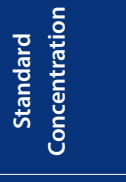 & $\overleftarrow{z}$ & 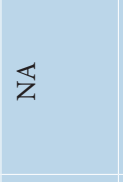 & $\stackrel{\breve{z}}{z}$ & & $\begin{array}{l}\vec{E} \\
\text { हू } \\
\text { है }\end{array}$ & $\stackrel{\mathbb{Z}}{\bar{z}}$ & $\stackrel{\vec{Z}}{\bar{z}}$ & 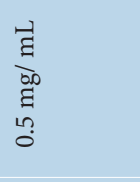 & & 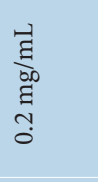 & & 로ํ. & $\begin{array}{l}\frac{\vec{\Xi}}{00} \\
\underline{\Xi}\end{array}$ & 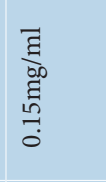 & \\
\hline 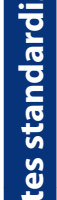 & 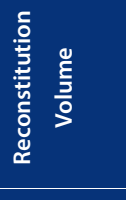 & $\overleftrightarrow{z}$ & $\overleftarrow{z}$ & 要 & & $\sum_{\infty}^{\bar{s}} \bar{g}$ & 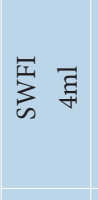 & $\begin{array}{l}\tilde{z} \\
\bar{Z} \\
\stackrel{0}{0} \\
\dot{1}\end{array}$ & $\stackrel{\bar{z}}{\bar{z}}$ & & 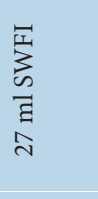 & & 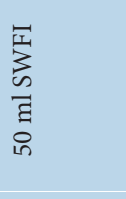 & $\stackrel{\grave{z}}{\bar{z}}$ & $\stackrel{\overleftarrow{Z}}{Z}$ & \\
\hline$\frac{\bar{g}}{20}$ & 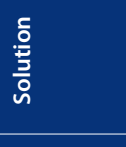 & $\begin{array}{l}3 \\
\text { Ln } \\
0 \\
\text { st }\end{array}$ & $\begin{array}{l}3 \\
1 \\
0 \\
n \\
z\end{array}$ & $\begin{array}{l}3 \\
\text { h } \\
0 \\
n \\
n\end{array}$ & & $\tilde{z}$ & $\tilde{z}$ & $\tilde{z}$ & $\begin{array}{l}3 \\
n \\
0 \\
n \\
n\end{array}$ & & $\begin{array}{l}3 \\
n \\
n \\
n \\
n\end{array}$ & & $\tilde{z}$ & $\tilde{z}$ & $\begin{array}{l}3 \\
\text { hn } \\
0 \\
\text { st }\end{array}$ & \\
\hline $\begin{array}{l}y \\
\frac{y}{7} \\
\frac{0}{0}\end{array}$ & 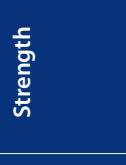 & 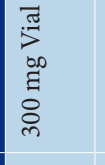 & 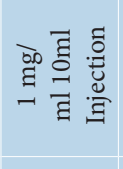 & 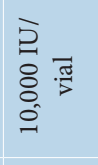 & & $\begin{array}{l}\text { हn } \\
8 \\
8\end{array}$ & $\begin{array}{l}\text { ह } \\
\text { है } \\
\text { है }\end{array}$ & $\sum_{\substack{n \\
2}}^{i n}$ & 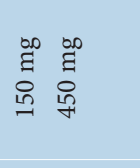 & & $\begin{array}{l}\stackrel{\infty}{\Xi} \\
\stackrel{\Xi}{\Xi}\end{array}$ & & 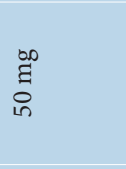 & $\stackrel{9}{\sim}$ & 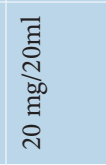 & \\
\hline 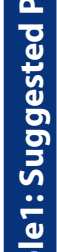 & 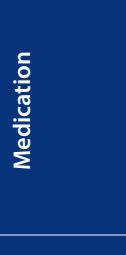 & 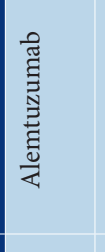 & 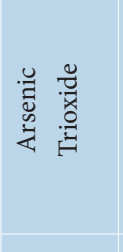 & 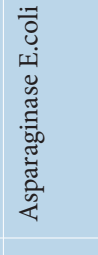 & & 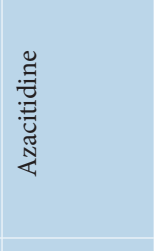 & 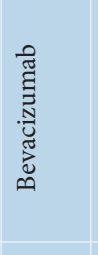 & 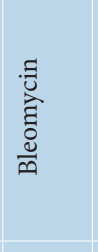 & 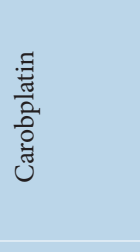 & & 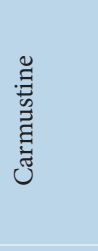 & & $\begin{array}{l}\frac{7}{2} \\
\frac{\pi}{20} \\
\frac{8}{3}\end{array}$ & 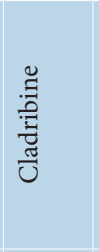 & 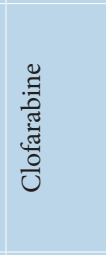 & \\
\hline & $\frac{0}{2}$ & - & $N$ & $m$ & & H & in & 6 & $n$ & & $\infty$ & & $a$ & $\stackrel{\ominus}{=}$ & $\exists$ & \\
\hline
\end{tabular}




\begin{tabular}{|c|c|c|c|c|}
\hline 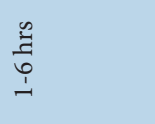 & $\stackrel{\infty}{\stackrel{2}{\leftrightarrows}}$ & $\begin{array}{l}\vec{\Xi} \\
\text { छ } \\
0\end{array}$ & $\begin{array}{l}\stackrel{\Xi}{\text { हี }} \\
8 \\
8\end{array}$ & 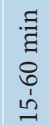 \\
\hline 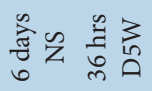 & 妾 & $\begin{array}{l}\stackrel{0}{\leftrightarrows} \\
\stackrel{\infty}{\infty}\end{array}$ & 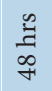 & $\overleftrightarrow{z}$ \\
\hline 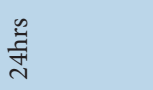 & 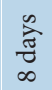 & $\begin{array}{l}\stackrel{\infty}{\Xi} \\
\stackrel{\sim}{*}\end{array}$ & $\frac{\infty}{己}$ & $\overleftrightarrow{Z}$ \\
\hline
\end{tabular}

\begin{tabular}{|c|c|c|c|c|c|c|c|c|c|}
\hline 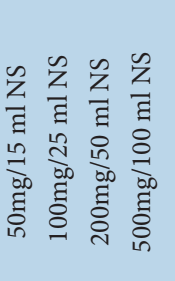 & 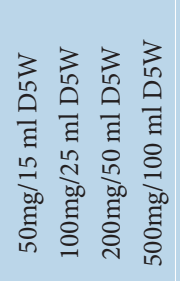 & 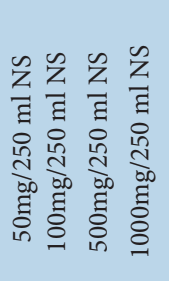 & 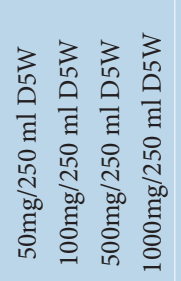 & 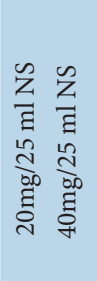 & 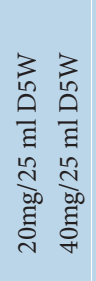 & 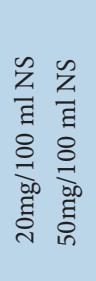 & 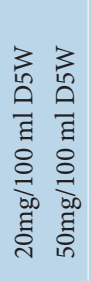 & 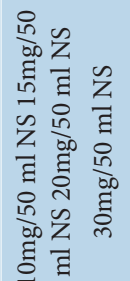 & 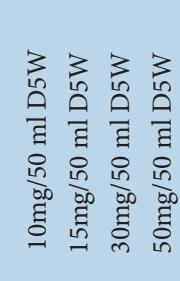 \\
\hline$\vec{\Xi}$ & & $\overleftarrow{z}$ & & $\overleftrightarrow{Z}$ & & 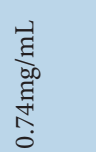 & & $\overleftrightarrow{z}$ & \\
\hline
\end{tabular}

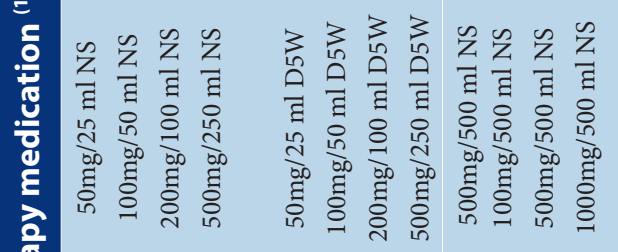
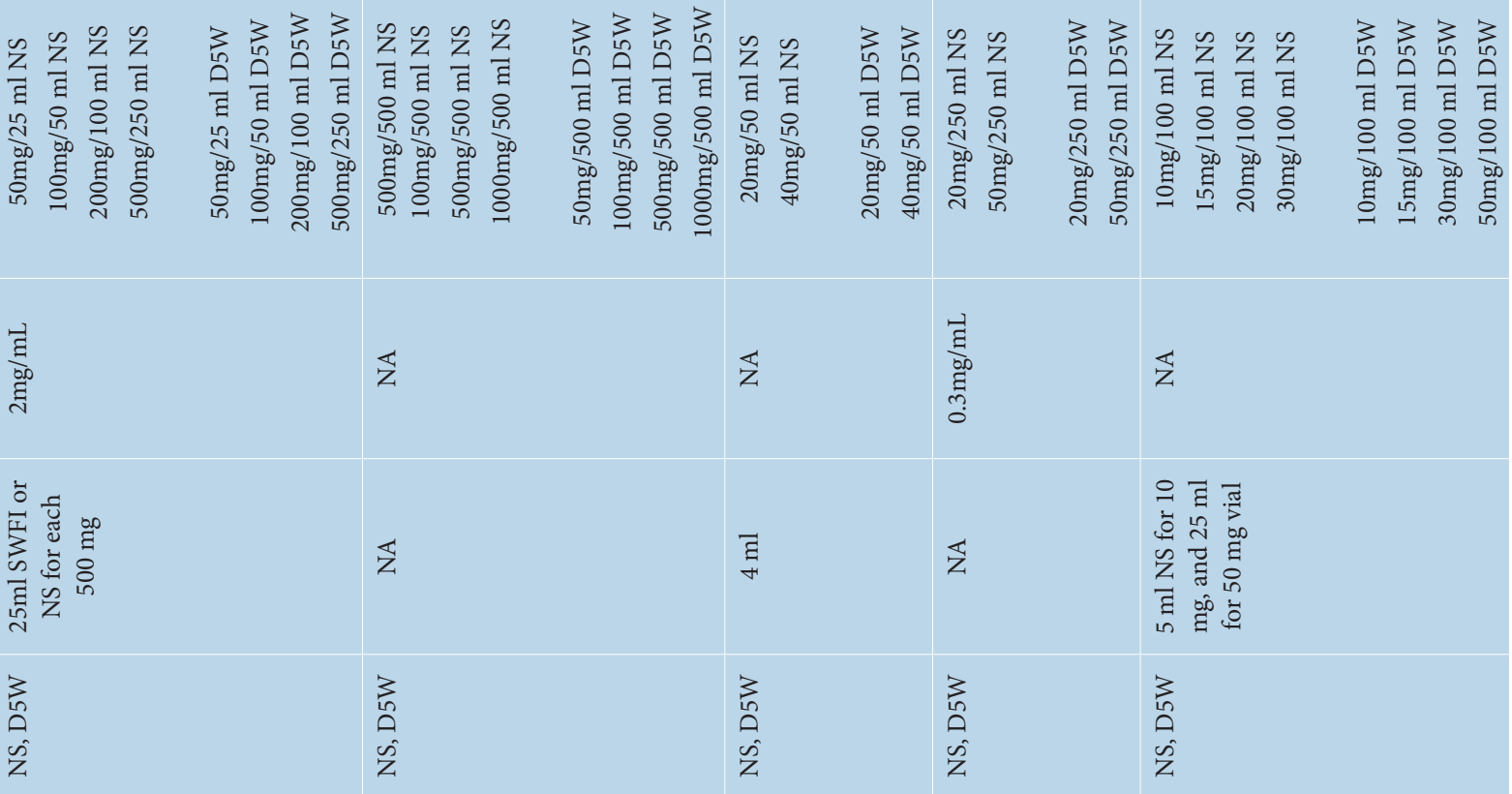

\begin{tabular}{|c|c|c|c|c|}
\hline 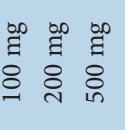 & 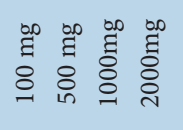 & 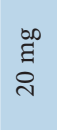 & 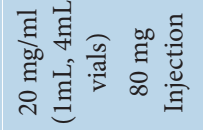 & $\begin{array}{ll}\infty & \infty \\
\vdots & \Xi \\
0 & 0 \\
0 & \text { in }\end{array}$ \\
\hline 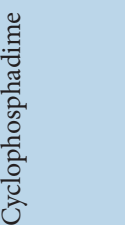 & 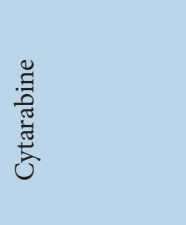 & 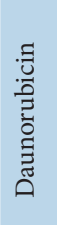 & 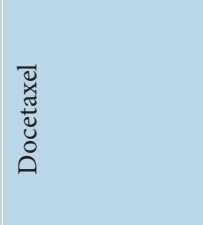 & 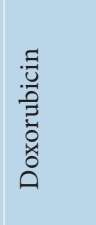 \\
\hline$\simeq$ & 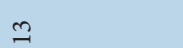 & $\Xi$ & $\stackrel{2}{2}$ & $\stackrel{0}{\circ}$ \\
\hline
\end{tabular}




\begin{tabular}{|c|c|c|c|c|c|c|c|c|}
\hline $\begin{array}{l}\text { 节 } \\
\text { 品 }\end{array}$ & $\begin{array}{l}\text { E् } \\
\text { ह } \\
\text { 品 }\end{array}$ & 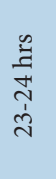 & 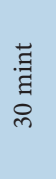 & 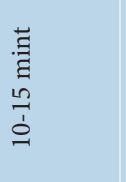 & $\begin{array}{l}\frac{0}{3} \\
\vdots \\
\vdots\end{array}$ & $\begin{array}{l}\text { 泀 } \\
\text { 号 }\end{array}$ & 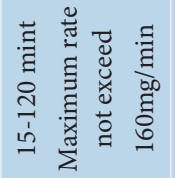 & 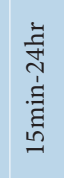 \\
\hline z' & $\begin{array}{l}\stackrel{\mathscr{n}}{\exists} \\
\underset{J}{J}\end{array}$ & 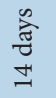 & $\mathbb{Z}$ & $\overleftrightarrow{z}$ & $\begin{array}{l}\stackrel{\infty}{\Xi} \\
\underset{\sim}{\sharp}\end{array}$ & 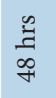 & $\stackrel{\vec{J}}{\vec{N}}$ & $\begin{array}{l}\stackrel{0}{3} \\
\substack{\infty \\
+}\end{array}$ \\
\hline 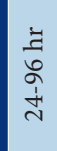 & 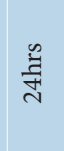 & 胥 & $\begin{array}{l}\stackrel{0}{\vec{Z}} \\
\underset{\sim}{\vec{N}}\end{array}$ & 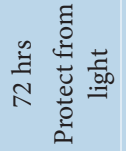 & $\overleftrightarrow{z}$ & $\stackrel{\mathscr{\Xi}}{\stackrel{\overbrace{}}{\sim}}$ & 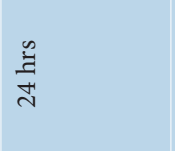 & 离 \\
\hline
\end{tabular}

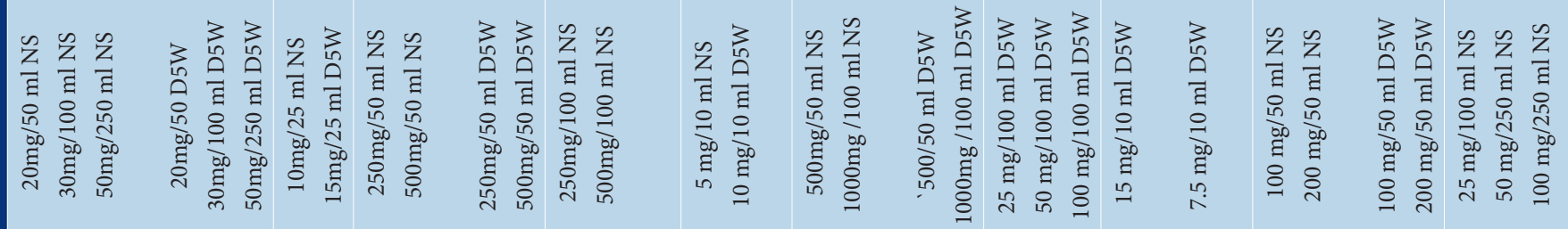
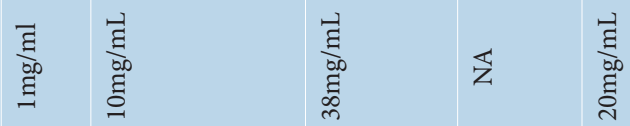

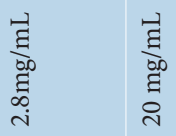

$\frac{\text { है }}{\text { है }}$

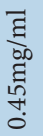
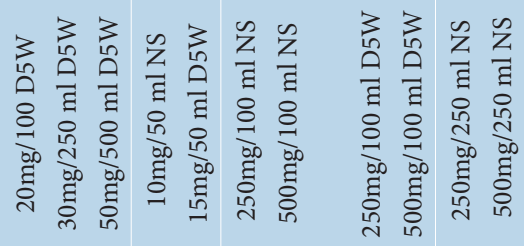

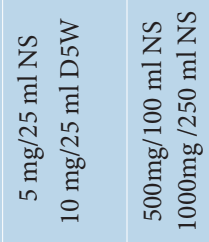
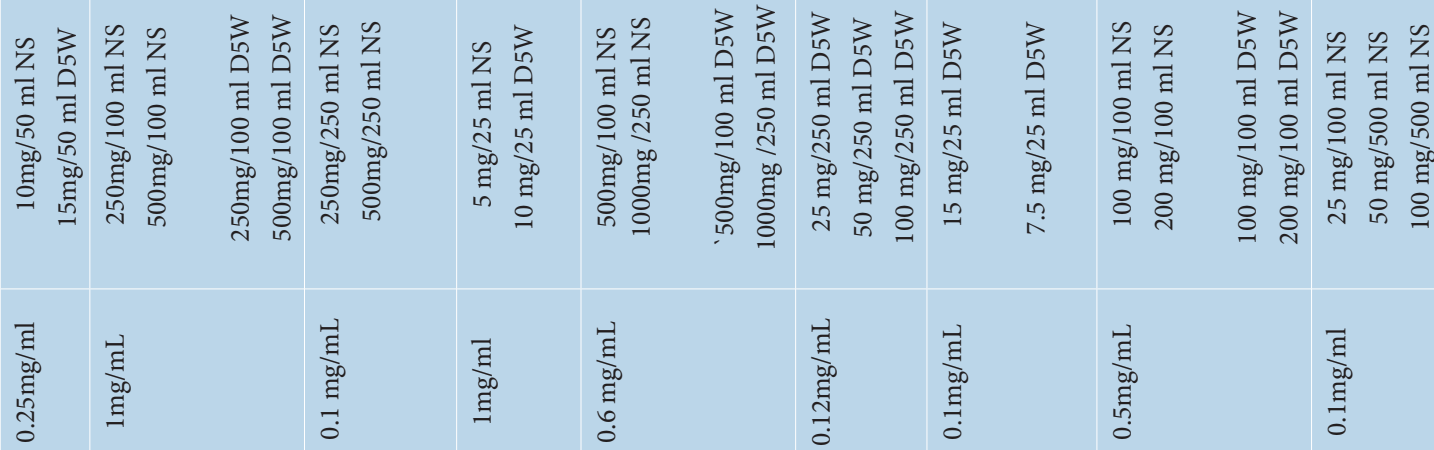

$\bar{\Xi}$
$\vdots$
$\vdots$
0
0

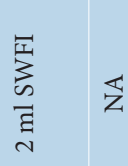

:

艺

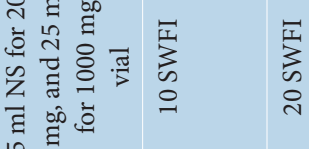

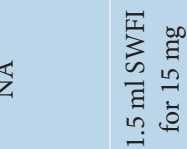

$\overleftrightarrow{Z}$

$\frac{n}{z}$

$\begin{array}{ll}3 & 3 \\ n & n \\ n & n \\ n & n\end{array}$

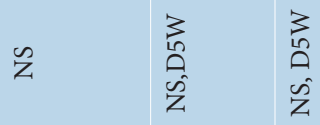

瓷

3
in
in

$\tilde{z}$

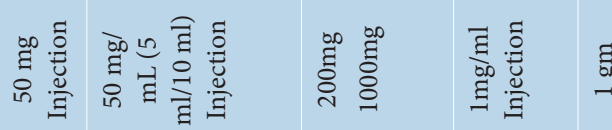

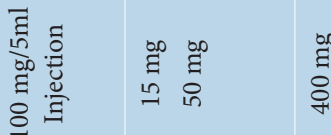

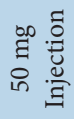

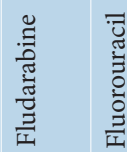

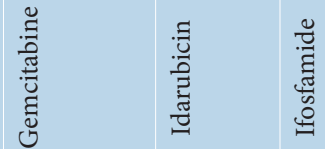

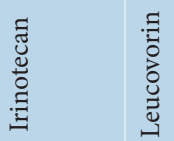

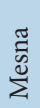

$\frac{\text { जี }}{\frac{\text { है }}{2}}$

$\stackrel{\infty}{\rightarrow}$

a

ำ

ㄱ

ข

ก

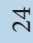

$\stackrel{12}{1}$

¿ 


\begin{tabular}{|c|c|c|c|c|c|c|c|c|c|c|c|c|}
\hline$\frac{\mathscr{m}}{\pi}$ & & $\begin{array}{l}\text { 音 } \\
\text { है } \\
\text { in }\end{array}$ & 袁 & & 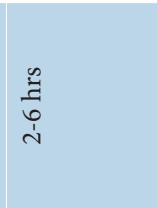 & 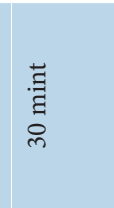 & & $\stackrel{\frac{\mathscr{m}}{7}}{\underset{I}{I}}$ & & 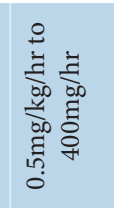 & & 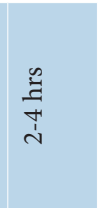 \\
\hline 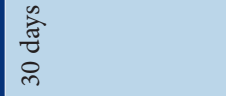 & & 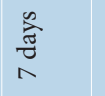 & $\underset{\substack{\pi\\
}}{\pi}$ & & $\frac{\substack{7\\
}}{d}$ & $\frac{7}{5}$ & & $\frac{y}{7}$ & & $\frac{\breve{z}}{\pi}$ & & $\frac{\mathscr{m}}{7}$ \\
\hline 䆑 & & 苟 & $\frac{\mathscr{m}}{\infty}$ & & $\frac{\mathscr{8}}{6}$ & $\frac{z}{6}$ & & 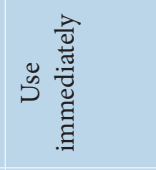 & & $\stackrel{\frac{Z}{\pi}}{\pi}$ & & $\frac{\mathscr{Q}}{\vec{z}}$ \\
\hline 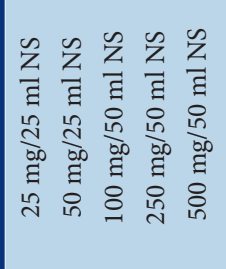 & 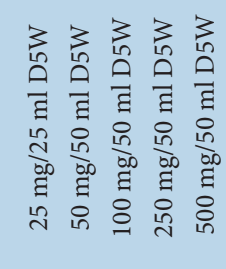 & 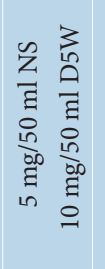 & 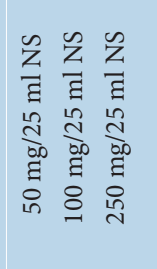 & 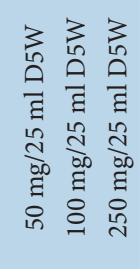 & 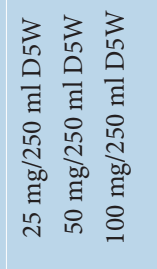 & 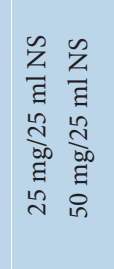 & 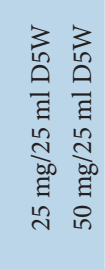 & 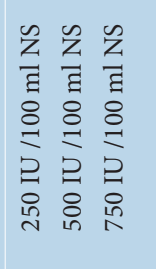 & 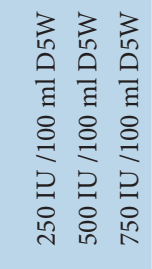 & 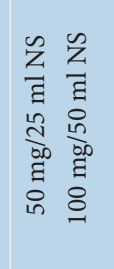 & 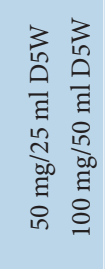 & 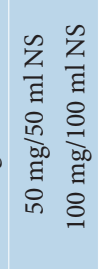 \\
\hline 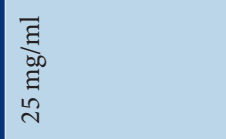 & & $\vec{z}$ & $\begin{array}{l}\bar{\Xi} \\
\stackrel{\Xi}{00} \\
\stackrel{\Xi}{\Xi}\end{array}$ & & $\overleftrightarrow{z}$ & 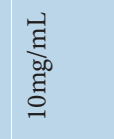 & & $\overleftrightarrow{z}$ & & 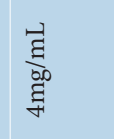 & & $\begin{array}{l}\vec{\Xi} \\
\stackrel{\vec{g}}{\mathrm{~b}} \\
\underline{E}\end{array}$ \\
\hline 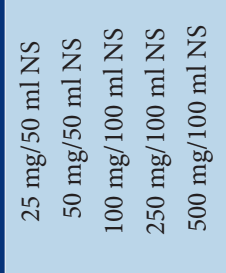 & 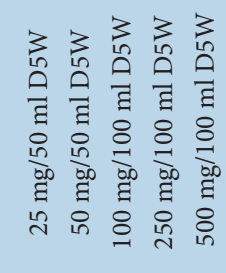 & 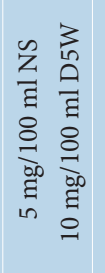 & 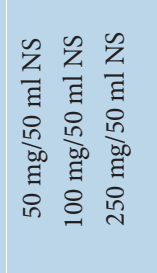 & 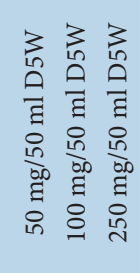 & 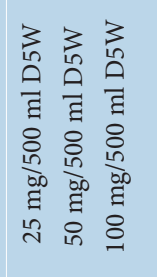 & 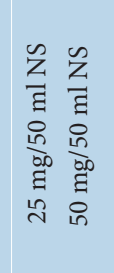 & 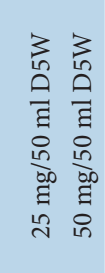 & 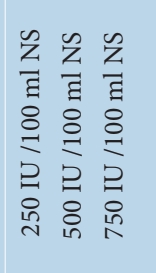 & 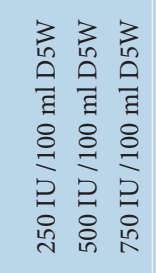 & 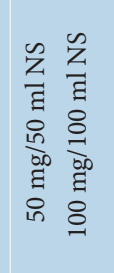 & 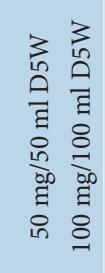 & 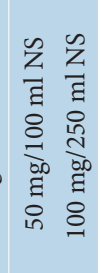 \\
\hline 艺 & & $\mathbb{z}$ & 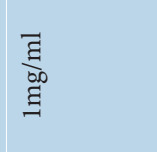 & & 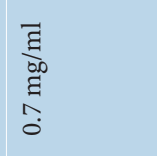 & $\begin{array}{l}\overrightarrow{\mathrm{E}} \\
\underline{\mathrm{o}} \\
\underline{\Xi}\end{array}$ & & $\overleftarrow{z}$ & & $\begin{array}{l}\overrightarrow{\underline{E}} \\
\overrightarrow{\underline{g}} \\
\underline{\Xi}\end{array}$ & & 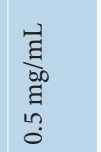 \\
\hline 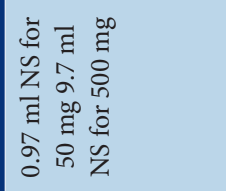 & & $\stackrel{\pi}{z}$ & $\mathbb{z}$ & & 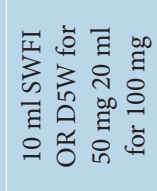 & 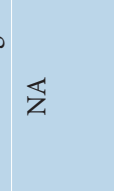 & & $\frac{1}{z}$ & & & & $\begin{array}{l}\text { च } \\
\sum_{0} \\
\overrightarrow{0} \\
\text { ह } \\
0\end{array}$ \\
\hline 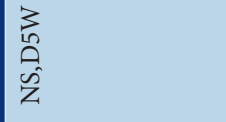 & & 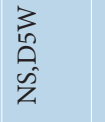 & 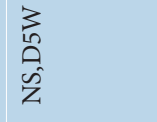 & & 瓷 & 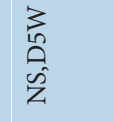 & & $\begin{array}{l}3 \\
m \\
0 \\
\text { s. }\end{array}$ & & 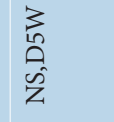 & & $\tilde{z}$ \\
\hline 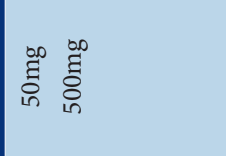 & & 高 & 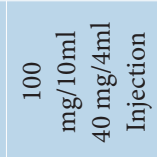 & & 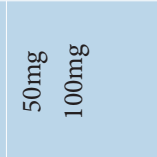 & 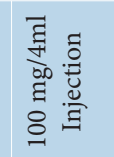 & & 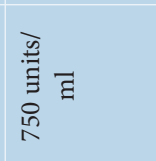 & & 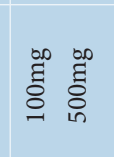 & & 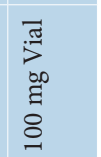 \\
\hline 跣 & & 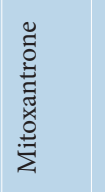 & 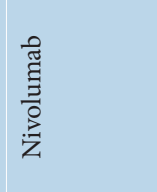 & & 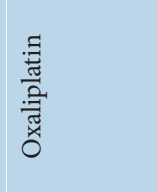 & 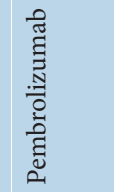 & & 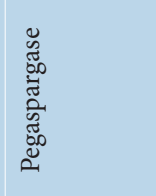 & & 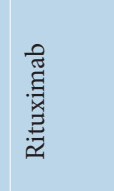 & & 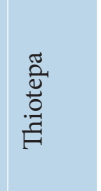 \\
\hline & & $\underset{\sim}{\infty}$ & ลิ & & 周 & $\vec{m}$ & & $\tilde{m}$ & & $\tilde{m}$ & & $\infty$ \\
\hline
\end{tabular}




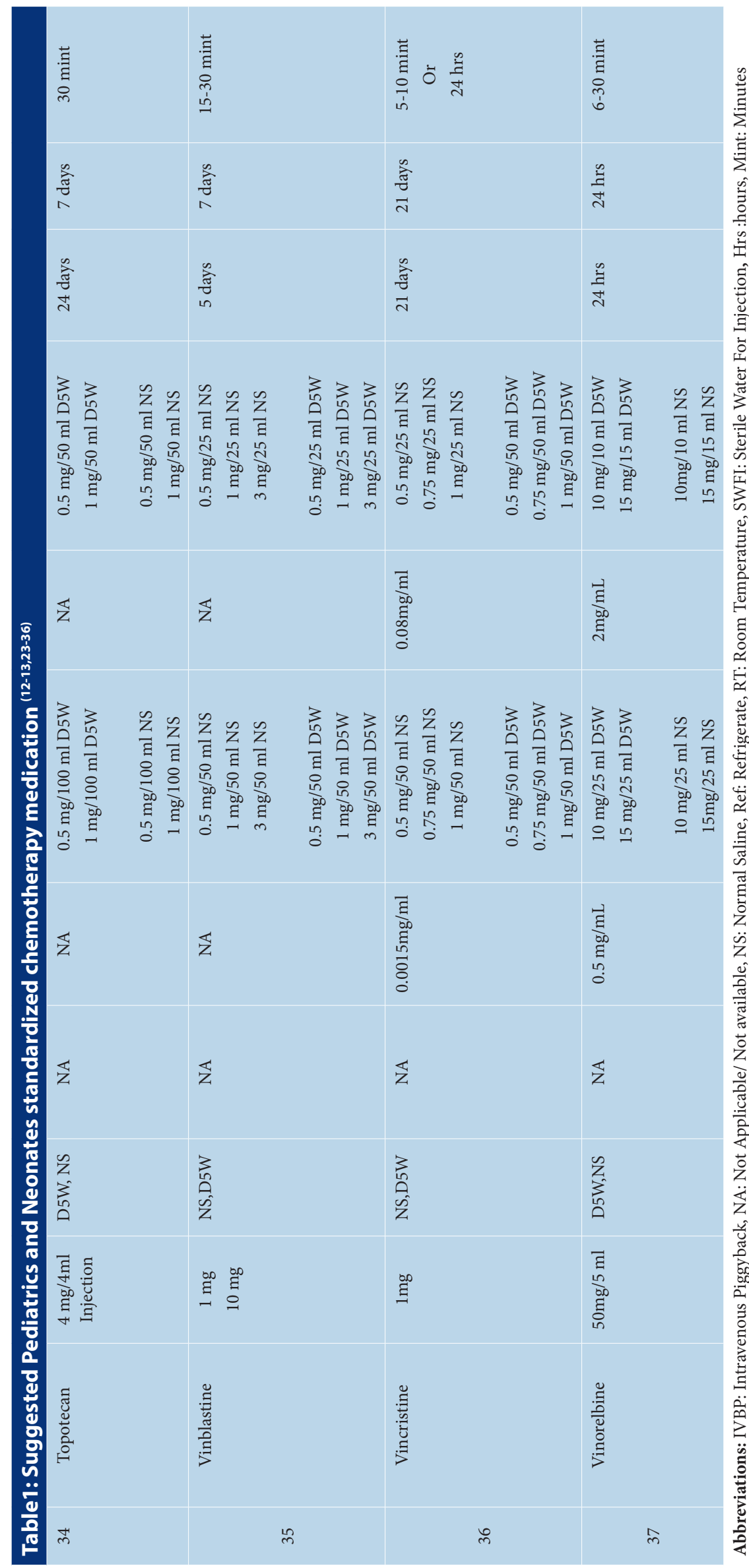

for any new healthcare or pharmacy staff joined the organization.

\section{Monitoring and Controlling Phase Project}

\section{Total Quality Management}

The Balance Scored Card is one of the total quantity management tools used for new project pediatrics and neonatal standardized concentration of chemotherapy medications.

BSC consisted of four types the customer, finance, internal process, education, and innovation. ${ }^{20}$ The declared example of internal processes was the assessment of healthcare services of patients and neonatal standardized concentration of chemotherapy medications. The type of education and innovation measured the clinical outcome of pediatrics and neonatal standardized concentration of chemotherapy medications and also explored the education and competency of pharmacy staff. Another example related to the financial type; the measurement of the economic impact of pediatrics and neonatal standardized concentration of chemotherapy medications, while the customer types may be measure the patients, pharmacy staff, healthcare professionals' pediatrics and neonatal standardized concentration of chemotherapy medications gratification in Saudi Arabia.

\section{Risk Management}

There are six types of risk management, for instance, the budget risks, scope risks, schedule risks, personal risks, technical risks and quality risks. ${ }^{[21,22]}$ The project might have exposed to typical risks such as personnel, budget, technical and quality risks. The current project suffered from personal risks related to the shortage of pharmacists or pharmacy technicians or not trained pharmacy staff. The second risk might be exposed to a financial budget risk; for example, the budget is not adequately covered the education and training and also not applicable to updated references. Also, it does not implement the computerized system during prescribing or alerting as it is another type of technical risk of the current project. The pediatrics and neonatal standardized chemotherapy concentration may be exposed to quality risks including of not fully implemented medication safety or non-quality pharmacist in the total management specialty. 


\section{Closing of the Project}

The pediatrics and neonatal standardized concentration of chemotherapy at all healthcare governmental and private organizations is highly suggested to prevent medication errors that might lead to mortality. Also, to avoid needless economic burden on hospitals and primary healthcare centers in the Kingdom of Saudi Arabia. The project should continue at chemotherapy IV admixture at each pharmacy services and related committees. The pediatrics and neonatal Education and training for standardized concentration should be done repeatedly, update drug concentration and expanded parental medications necessary in the future. The annual celebration of all pediatrics and neonatal pharmacist and pharmacy technician staff is highly optional in the Kingdom of Saudi Arabia.

\section{ACKNOWLEDGEMENT}

None.

\section{CONFLICT OF INTEREST}

None.

\section{FUNDING}

None

\section{CONSENT FOR PUBLICATIONS}

Informed consent was obtained from all the participants

\section{ETHICAL APPROVAL}

This research exempted from research and ethical committee or an institutional review board (IRB) approval.

https://www.hhs.gov/ohrp/regulations-andpolicy/decision-charts-2018/index.html

\section{ABBREVIATIONS}

MOH: Ministry of Health; KSA: Kingdom of Saudi Arabia; TPN: Total Parenteral Nutrition; SWOT: Strengths, Weaknesses, Opportunities and Threats; IV: Intravenous; BSC: Balance Scored Cards; IAC: intravenous admixture committee.

\section{ORCID ID}

Yousef Ahmed Alomi (D) https://orcid.
org/0000-0003-1381-628X

\section{REFERENCES}

1. Alomi YA. National medication safety program at ministry of health in Saudi Arabia. J Pharmacovigil. 2015;3(5):e145.

2. Alomi YA, Alghamdi SJ, Alattyh RA. Strategic plan of general administration of pharmaceutical care at ministry of health in Saudi Arabia 2012-2022. J Pharm Pharm Scien. 2015;1(13):1-8.

3. Alanazi1 AA, Alomi YA, Almaznai MM, et al. Pharmacist's intervention and medication errors prevention at pediatrics, obstetrics and gynecology hospital in East Province, Saudi Arabia. Int $J$ Pharm Heal Sci. 2019;2(2):122-8.

4. Alomi YA, Alanazi AA, Almaznai MM, et al. Costeffectiveness analysis of medication safety program at pediatrics, obstetrics and gynecology hospital, East Province, Saudi Arabia. Pharmacol Toxicol Biomed Reports. 2019;5(3s):S12-6.

5. Alomi1 YA, Fallatah AO, Al-Shubaar N, et al. The clinical outcomes of pharmacist interventions in total parenteral nutrition services in Riyadh City, Saudi Arabia. Int J Pharm Heal Sci. 2019;2(2):13540.

6. Alomi YA, Fallatah AO, Bahadig FA, et al. The economic outcomes of pharmacist interventions in total parenteral nutrition services in Saudi Arabia. Pharmacol Toxicol Biomed Reports. 2019;5(3s):S40-9

7. Ahmed AY, Saad AH, Fallatah AO, et al. Neonatal Total Parenteral Nutrition: Initiative and implementation of standardized formulation in Saudi Arabia. Res Pharm Heal Sci. 2018;4(3):492-6.

8. Ahmed AY, Saad AH, Fallatah AO, et al. Pediatrics' total parenteral nutrition: Initiative and implementation of standardized formulation in Saudi Arabia. Res Pharm Heal Sci. 2018;4(3):492-6.

9. United States Pharmacopeia. USP General Chapter - Hazardous Drugs- Handling in Healthcare Settings. The United States Pharmacopeial Convention. 2017;1-20.

10. Goldspiel B, Hoffman JM, Griffith NL, et al. ASHP guidelines on preventing medication errors with chemotherapy and biotherapy. Am J Health Syst Pharm. 2015;72(8):e6-35.

11. Murray KL, Wright D, Laxton B, et al. Implementation of standardized pediatric i.v. medication concentrations. Am J Heal Pharm. 2014;71(17):15008.

12. Benizri $F$, Bonan $B$, Ferrio $A L$, et al. Stability of antineoplastic agents in use for home-based intravenous chemotherapy. Pharm World Sci. 2009;31(1):1-13

13. Vigneron J, Astier A, Hecq JD, et al. SFPO and ESOP recommendations for the practical stability of anticancer drugs: An update. Eur J Oncol Pharm. 2014;8(2):3-13.

14. Institute for Safe Medication Practices (ISMP). Standard Concentrations of Neonatal Drug Infusions. ISMP. 2011.

15. Alomi YA. National Intravenous (IV) Therapy Program at $\mathrm{MOH}$ in Saudi Arabia. EC Pharm Sci. 2016;3(2 and 3):307-11.

16. McDonough R. Writing a business plan for a new pharmacy service. The Dynamics of Pharmaceutical Care: Enriching Patients' Health. 2010;23:1-2.

17. Harris IM, Baker E, Berry TM, et al. Developing a business-practice model for pharmacy ser vices in ambulatory settings. Pharmacotherapy. 2008;28(2):7e-34e
18. Sachdev G. Sustainable business models: Systematic approach toward successful ambulatory care pharmacy practice. Am J Heal Pharm. 2014;71(16):1366-74.

19. PMBOK Guide. A guide to the project management body of knowledge. Sixth Edition. Project Management Institute Inc. 2017.

20. Kaplan RS, Norton DP. The balanced scorecard: Measures that drive performance. Harvard Business Review. 2005;83(7):172. Cited 2020 Mar 15. Available from: https://hbr.org/1992/01/thebalanced-scorecard-measures-that-drive-performance-2.

21. Ray S. The risk management process in project management - Project Manager. Risk Management. 2017. Cited 2020 Mar 15. Available from: https://www.projectmanager.com/blog/risk-management-process-steps.

22. Kaplan RS, Mikes A. Managing risks: A new framework. Harvard Business Review. 2012:90(6):4860. Cited 2020 Mar 15. Available from: https://hbr. org/2012/06/managing-risks-a-new-framework.

23. Brown P. Pediatric acute lymphoblastic leukemia. NCCN Clinical Practice Guidelines in Oncology. 2019:2:1-118.

24. Davies K. Pediatric Aggressive Mature B-Cell Lymphomas. NCCN Clinical Practice Guidelines in Oncology. 2019;1:1-35.

25. Adams SC, Vyas HM, Anderson RW. Pharmaceutical issues in infusion chemotherapy stability and compatibility. In: Cancer Chemotherapy by Infusion. 1987;100-13.

26. Ministry of Health. Ministry of Health Formulary. Health Ministry of Health. 2012

27. Saudi Food and Drug Authority. List of human medicine and herbal health. 2019. [cited 2019 Jun 17]. Available from: https://www.sfda.gov.sa/en/ drug/resources/Pages/DrugsUnderRegistrations. aspx

28. Baxter K, Aikman K, Luckhurst R, et al. British National Formulary 78 (BNF). Royal Phamaceutical Society. 2019;1-1701.

29. Alomi YA, et al. Pediatrics Parenteral Dilution Manual. Ministry of Health. 2015. Available from: https://www.researchgate.net/publication/281710402_Pediatrics_Parenteral_Dilution_ Manual

30. Alomi YA, et al. Neonates Parenteral Dilution Manual. Ministry of Health. 2015. Available from: https://www.researchgate.net/publication/281710411_Neonates_Parenteral_Dilution_ Manual

31. Wolters Kluwer Clinical Drug Information. Inc. (Lexi-Drugs). Wolters Kluwer Clinical Drug Information, Inc. 2020.

32. Drugs.com. Drugs.com, Prescription Drug Information, Interactions and Side Effects. Drugs.com. 2020. [cited 2020 Jun 16]. Available from: https:// www.drugs.com/

33. King Abdulaziz Medical City. Unified IV Manual. 2020.

34. Wolters Kluwer Clinical Drug Information. Inc. (ASHF Essentials adults and pediatrics). Wolters Kluwer Clinical Drug Information, Inc. 2020.

35. Wolters Kluwer Clinical Drug Information. Inc. (Pediatrics and Neonatal Lexi-Drugs). Wolters Kluwer Clinical Drug Information, Inc. 2020.

36. Wolters Kluwer Clinical Drug Information. Inc. (Nursing Lexi-Drug). Wolters Kluwer Clinical Drug Information, Inc. 2020. 